\title{
A 6-month open-label extension study of the safety and efficacy of subcutaneous belimumab in patients with systemic lupus erythematosus
}

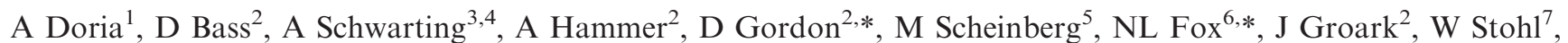 \\ C Kleoudis ${ }^{8}$ and D Roth ${ }^{2}$ \\ ${ }^{1}$ Rheumatology Unit, Department of Medicine, University of Padova, Padua, Italy; ${ }^{2}$ GlaxoSmithKline R\&D, Philadelphia, PA, USA; ${ }^{3}$ Division of \\ Rheumatology, University Hospital Mainz, Mainz, Germany; ${ }^{4}$ ACURA Rheumazentrum Kliniken, Bad Kreuznach, Germany; ${ }^{5}$ Centro de \\ Pesquisas Clinicas do Hospital Abreu Sodré, São Paulo, Brazil; ${ }^{6}$ GlaxoSmithKline R\&D, Rockville, MD, USA; ${ }^{7}$ Division of Rheumatology, \\ University of Southern California Keck School of Medicine, Los Angeles, CA, USA; and ${ }^{8}$ Parexel Clinical Research, Raleigh-Durham, NC, USA
}

\begin{abstract}
Objective: To evaluate the safety, tolerability and efficacy of subcutaneous (SC) belimumab in patients with systemic lupus erythematosus (SLE) beyond 1 year. Methods: This was a 24week, open-label extension following a 52-week, double-blind, placebo-controlled trial of belimumab SC. Patients who completed the double-blind phase were eligible to enter the open-label phase. All patients received weekly belimumab $200 \mathrm{mg}$ SC plus standard SLE therapy. Outcome measures included safety and efficacy (SLE Response Index (SRI) and SLE Flare Index (SFI) rates), and changes in biomarker and B cell levels. Results: Of 677 patients who completed the 52-week, double-blind phase, 662 entered the open-label phase; 206 had previously received placebo and 456 had previously received belimumab. Despite differences in total belimumab exposure (24 weeks in the placebo-to-belimumab group versus 76 weeks in the belimumab group), the proportions of patients experiencing more than one adverse event (AE) or a serious AE in the open-label phase were similar between groups (placebo-to-belimumab: 51.5 and 6.8\%; belimumab: 48.2 and 5.5\%, respectively). Most AEs were mild/moderate in severity. Efficacy was maintained through the extension phase. An SRI response was achieved by $16.1 \%$ of patients in the placebo-to-belimumab group and $76.3 \%$ patients in the belimumab group. Furthermore, $1.0 \%$ of patients in the placebo-to-belimumab group and $2.6 \%$ of patients in the belimumab group experienced a severe SFI flare. Conclusion: Belimumab SC was well tolerated and efficacy was maintained during the extension phase of this study. The safety profile of belimumab SC is consistent with that of previous experience with belimumab. Trial registration: ClinicalTrials.gov identifier: NCT01484496 Lupus (2018) 27, 1489-1498.
\end{abstract}

Key words: Belimumab; B-lymphocyte stimulator; corticosteroids; open-label extension; SLE responder index; subcutaneous; systemic lupus erythematosus

\section{Introduction}

Belimumab is a human monoclonal antibody that binds to and inhibits the biological activity of Blymphocyte stimulator, ${ }^{1}$ a potent B cell survival factor associated with human systemic lupus

\footnotetext{
*Affiliated to the respective institutions at the time of study.
}

Correspondence to: Andrea Doria, Rheumatology Unit, Department of Medicine, University of Padova, Via Giustiniani 2, 35128, Padua, Italy.

Email: adoria@unipd.it

Received 9 January 2018; accepted 23 April 2018

(c) The Author(s), 2018. Reprints and permissions: http://www.sagepub.co.uk/journalsPermissions.nav erythematosus (SLE) disease. ${ }^{2}$ Intravenous (IV) belimumab $10 \mathrm{mg} / \mathrm{kg}$ was the first, and to date only, biological drug to be approved for the treatment of adult patients with active, autoantibodypositive SLE. $^{3-5}$ Since its approval, evidence from clinical practice has demonstrated that belimumab is associated with improvements in control of disease activity, reductions in fatigue and improvements in ability to work. ${ }^{6}$ Therefore, belimumab is cost-effective both in terms of employment and in decreasing health resource utilization. ${ }^{6}$

Although the approval of belimumab IV was a significant step in improving treatment options for patients with SLE, the IV administration route can 
pose challenges for some patients. Belimumab IV must be administered at a clinic or infusion centre, which is resource-intensive for the healthcare system and time-consuming for patients. Hence, to enhance usability, a new subcutaneous (SC) formulation of belimumab has been developed. ${ }^{7}$ Comparison of weekly dosing of belimumab $200 \mathrm{mg}$ SC with belimu$\mathrm{mab} 10 \mathrm{mg} / \mathrm{kg}$ IV administered every 4 weeks demonstrated that the dosing regimens result in similar exposure. ${ }^{8,9}$ Belimumab SC administration may provide greater convenience to patients versus IV administration due to its less invasive and quicker administration time. Moreover, it can be administered outside of the doctor's clinic by patients or their caregivers. These advantages of SC administration are particularly important for the chronic longterm treatment of SLE. ${ }^{10-12}$

A study of belimumab administered subcutaneously in subjects with SLE (BLISS-SC) (GlaxoSmithKline [GSK] study BEL112341; NCT01484496), a 52-week Phase III, double-blind study, demonstrated the efficacy and safety of belimumab SC delivered via a prefilled syringe in patients with active, autoantibody-positive SLE. Weekly doses of belimumab $200 \mathrm{mg}$ SC plus standard SLE therapy (SoC) significantly improved the SLE Response Index 4 (SRI4) response and reduced the incidence of severe flares compared with placebo. Moreover, belimumab was generally well tolerated and the rates of adverse events (AEs) were similar across belimumab and placebo groups. ${ }^{7}$ Here, we present the results of a 6month extension to BLISS-SC, conducted to evaluate the safety, tolerability and efficacy of belimumab SC in patients with SLE who completed the 52-week parent study.

\section{Methods}

\section{Study design}

This was a 24-week multicentre, open-label extension phase of the BLISS-SC study (GSK study BEL112341; NCT01484496), conducted between November 2011 and October 2015 at 177 sites in 30 countries across North America, Central America, South America, Western Europe, Eastern Europe and Asia. ${ }^{7}$ Details of the BLISSSC study design have been reported previously. ${ }^{7}$

\section{Patients}

Patient inclusion and exclusion criteria for the BLISS-SC study have been described previously. ${ }^{7}$
In brief, at screening, patients were $\geq 18$ years of age with a diagnosis of SLE classified according to the American College of Rheumatology criteria, ${ }^{13}$ a Safety of Estrogens in Lupus Erythematosus National Assessment-Systemic Lupus Erythematosus Disease Activity Index (SELENA-SLEDAI) score $\geq 8$ and were antinuclear antibody and/or anti-double-stranded DNA (dsDNA)positive. ${ }^{7}$ Patients with severe lupus kidney disease or severe central nervous system lupus were excluded. Patients who completed the 52-week double-blind phase were eligible to participate in the open-label extension phase. ${ }^{7}$ Patients who had previously received placebo in the double-blind phase were switched to belimumab $200 \mathrm{mg}$ SC weekly in the extension phase (placebo-to-belimumab group); patients who had previously received belimumab $200 \mathrm{mg}$ SC continued to receive the same dose (belimumab group) (Figure 1). Patients received the first dose of belimumab approximately 1 week after the last dose in the double-blind phase of the study. All patients received SoC throughout the study.

All patients provided written informed consent prior to enrolment. Ethics committee/institutional review board approval was obtained for all study sites. The study was conducted in accordance with the ethical principles of the Declaration of Helsinki 2008, the International Council for Harmonisation on Good Clinical Practice and the applicable country-specific regulatory requirements. ${ }^{14}$

\section{Endpoints and assessments}

Safety

The safety of belimumab was evaluated by monitoring AEs, serious AEs (SAEs), AEs of special interest (AESIs; malignancies, post-injection systemic reactions, infections, depression/suicide/self-injury and deaths), clinical laboratory tests (haematology, chemistry and routine urinalysis) and immunogenicity throughout the 24-week extension phase. AEs were coded according to the Medical Dictionary for Regulatory Activities Version 18.

\section{Efficacy}

For all efficacy analyses, baseline was defined as the last assessment prior to the first dose of belimumab; for the belimumab group, this was day 0 of the double-blind phase and for the placebo-to-belimumab group, this was week 52 of the double-blind phase.

Efficacy was evaluated by SRI4 at week 24 and is defined as a $\geq 4$-point reduction from baseline in SELENA-SLEDAI score, no worsening (increase 


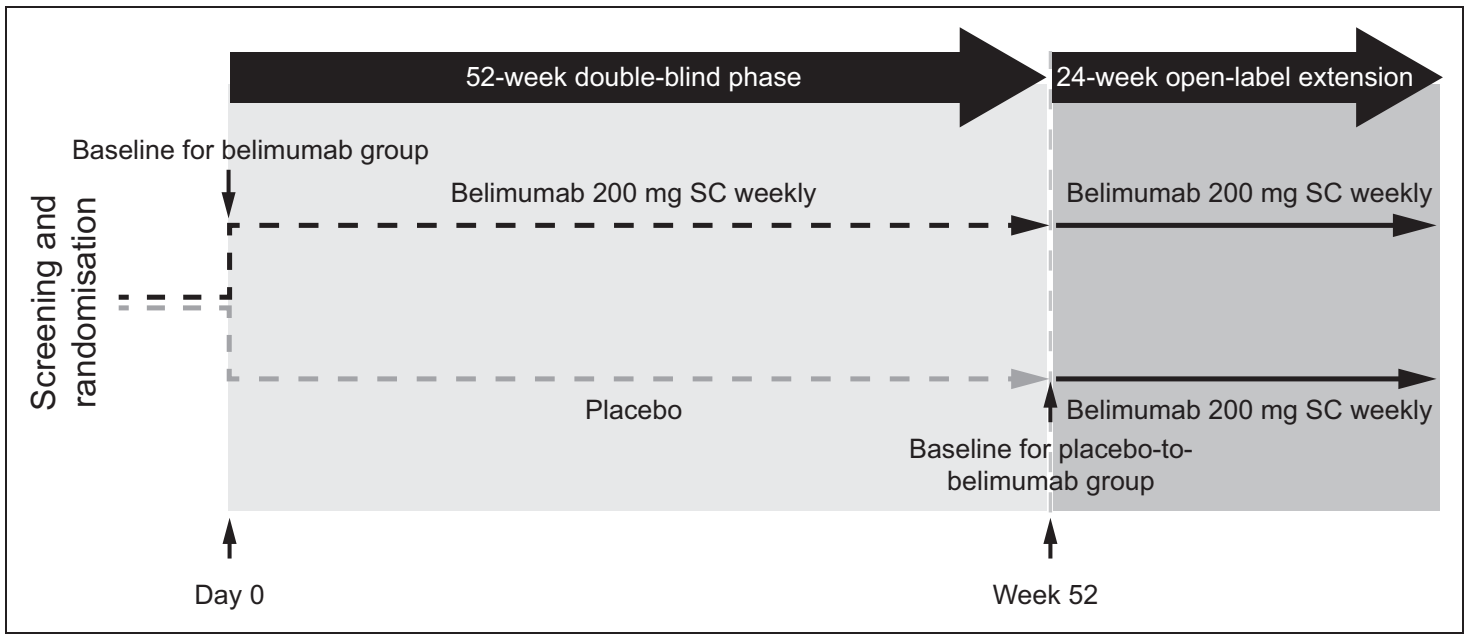

Figure 1 Study design of BLISS-SC trial and subsequent open-label extension phase.

BLISS-SC: A study of belimumab administered subcutaneously in subjects with systemic Lupus Erythematosus; SC: subcutaneous.

of $<0.30$ points from baseline) in Physician's Global Assessment (PGA), and no new British Isles Lupus Assessment Group (BILAG) A organ domain score or two new BILAG B organ domain scores compared with baseline. ${ }^{15}$ Other efficacy assessments included time to first severe SFI flare during the open-label phase, percentage of patients with daily prednisone dose reduced from $>7.5 \mathrm{mg}$ / day at baseline to $\leq 7.5 \mathrm{mg}$ /day at week 24 , percentage of patients with daily prednisone dose increased from $\leq 7.5 \mathrm{mg}$ /day at baseline to $>7.5 \mathrm{mg} /$ day at week 24 , time to first renal flare and time to first renal flare among patients with baseline proteinuria $>0.5 \mathrm{~g} / 24$ hours.

\section{Biomarkers}

Biomarker assessments included changes in antidsDNA, serum complement levels (C3 and C4) and mean percent change from baseline in $\mathrm{B}$ cell subsets (CD20+; CD19+/CD20+/CD27-naïve; CD19+/ $\mathrm{CD} 20+/ \mathrm{CD} 69+$ activated; CD19+/CD20+/CD27+ memory; CD19+/CD20+/CD138 + plasmacytoid; CD19+/CD20-/CD138 + plasma cells; CD19+/ CD38b+/CD27b lymph SLE subset; and CD19+/ CD24b+/CD38b+/CD27-transitional).

\section{Data analyses}

Enrolment in the open-label extension phase was dependent on the number of patients completing the 52-week parent study; therefore, no sample size calculations were performed.

All analyses were conducted in the intent-to-treat population, defined as all randomized patients who received at least one dose of belimumab in the extension phase.
No formal statistical hypothesis testing was performed for the open-label phase.

Continuous variables were summarized using mean, median, standard deviation (SD), 25th and 75th percentile, and minimum and maximum values. The analyses were performed using SAS software version 9.3 (SAS Institute Inc., Cary, $\mathrm{NC}$, USA).

\section{Results}

\section{Patient population}

Of 677 patients who completed the 52-week doubleblind phase, $662(97.8 \%)$ entered the open-label extension phase. Of these, 206 patients who had received placebo during the double-blind phase were switched to belimumab $200 \mathrm{mg}$ SC and 456 patients who had received belimumab $200 \mathrm{mg}$ SC during the double-blind phase continued to do so in the open-label extension phase (Figure 2). A total of $625(94.4 \%)$ patients completed the openlabel extension phase and $37(5.6 \%)$ withdrew. The most common reason for withdrawal was AEs, which were observed at a similar frequency in both treatment groups (placebo-to-belimumab: 2.4\%; belimumab: $2.9 \%$ ).

Baseline (last assessment prior to the first dose of belimumab) demographics were similar between treatment groups (Table 1); the majority of patients were female $(94.6 \% ; 626 / 662)$, with a mean (SD) age of 38.7 (11.86) years. Systemic Lupus International Collaborating Clinics/American College of Rheumatology Damage Index scores were similar between groups; however, patients 


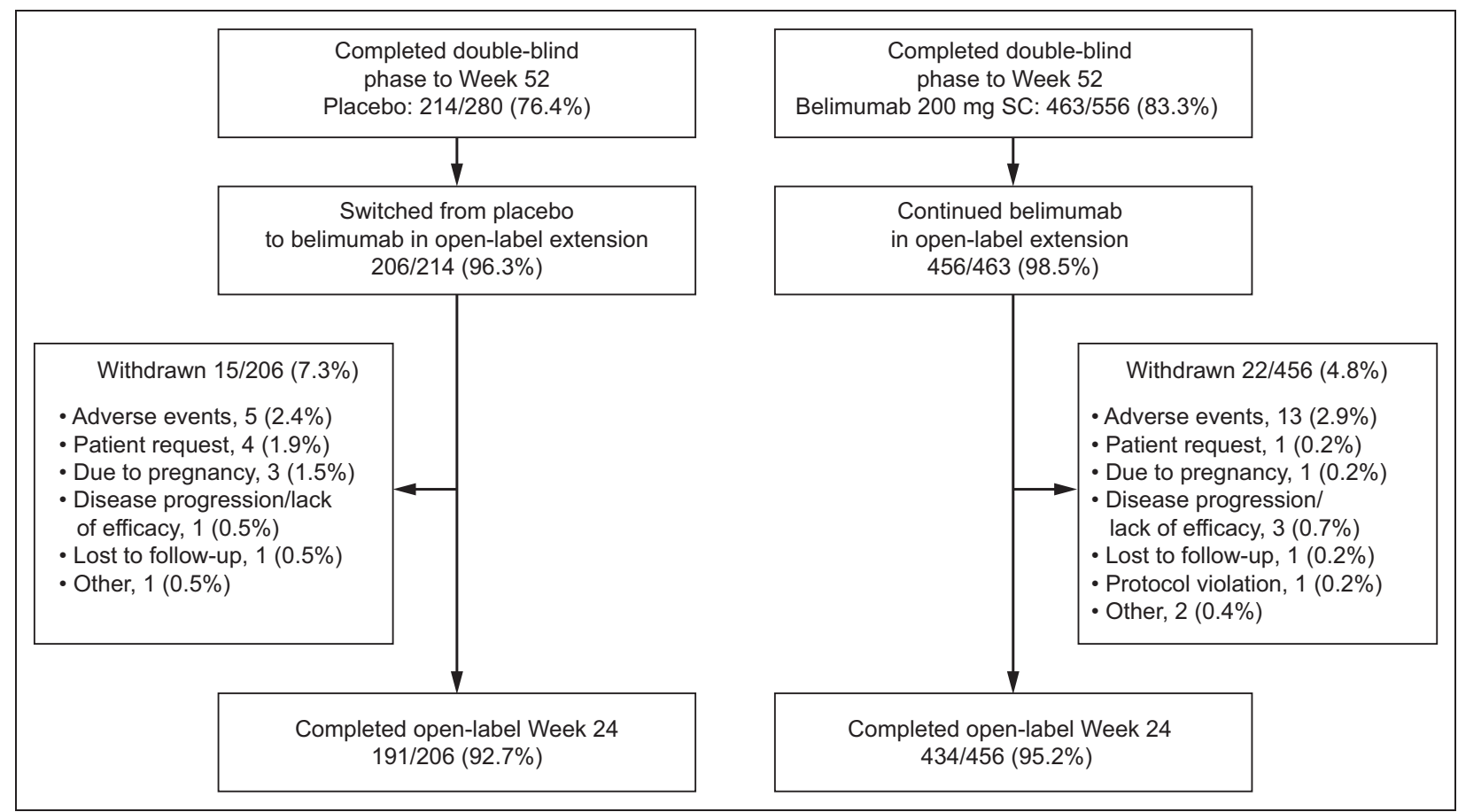

Figure 2 Patient disposition.

SC, subcutaneous.

in the placebo-to-belimumab group had lower BILAG, SELENA-SLEDAI and PGA scores compared with the belimumab group (Table 1). There were $55(26.7 \%)$ patients in the placebo-to-belimumab group who had a baseline SELENA-SLEDAI score $<4$ as a result of improvements that occurred between screening prior to the start of the doubleblind phase and baseline (week 52 of the doubleblind phase). Two $(0.4 \%)$ patients in the belimumab group had a baseline SELENA-SLEDAI score $<4$ due to fluctuations occurring between screening and baseline. Patients with a SELENASLEDAI score $<4$ at baseline were excluded from the SELENA-SLEDAI analyses.

\section{Safety}

\section{Adverse events}

Despite the differences in cumulative belimumab exposure (placebo-to-belimumab: 24-week openlabel extension only; belimumab: 52-week doubleblind phase plus 24-week open-label extension), the proportion of patients experiencing an $\mathrm{AE}$ during the 24-week open-label extension was similar between the treatment groups (placebo-to-belimumab: $51.5 \%$; belimumab: $48.2 \%$ ) (Table 2). The majority of AEs were mild to moderate in severity. Infections and infestations were the most frequent AEs by system organ class (placebo-to-belimumab: $29.6 \%$; belimumab: $28.3 \%$ ) (Table 2). SAEs occurred in $6.8 \%$ of patients in the placebo-to-belimumab group and $5.5 \%$ of patients in the belimumab group. The incidence of serious infections and infestations was $3.4 \%$ in the placebo-to-belimumab group compared with $2.2 \%$ in the belimumab group. AEs leading to discontinuation of belimumab occurred in $5(2.4 \%)$ patients in the placeboto-belimumab group and $12(2.6 \%)$ patients in the belimumab group.

Overall, the incidence of AESIs was low. The incidence of post-injection systemic reactions was $4.4 \%(9 / 206)$ in the placebo-to-belimumab group and $2.6 \%(12 / 456)$ in the belimumab group. The incidence of local injection-site reactions was $1.5 \%(3 / 206)$ in the placebo-to-belimumab group and $0.2 \%(1 / 456)$ in the belimumab group; none were serious or resulted in study discontinuation. Four $(1.9 \%)$ patients in the placebo-to-belimumab group and $8(1.8 \%)$ patients in the belimumab group experienced depression. One $(0.2 \%)$ patient in the belimumab group had a suicide attempt that did not result in death (Table 2).

Two deaths occurred in the open-label phase. Metabolic acidosis occurred in a 40-year-old male in the placebo-to-belimumab group who developed grade 4 SAEs of deep vein thrombosis, antiphospholipid syndrome, acute kidney injury and metabolic acidosis 31 days after receiving the first dose of belimumab $200 \mathrm{mg}$ SC. Grade 4 acute 
Table 1 Baseline patient demographics and characteristics (intent-to-treat population)

\begin{tabular}{|c|c|c|}
\hline Characteristic & $\begin{array}{l}\text { Placebo-to-belimumab } \\
200 \mathrm{mg} \mathrm{SC} \mathrm{n}=206\end{array}$ & $\begin{array}{l}\text { Belimumab } 200 \mathrm{mg} \\
\mathrm{SC} \mathrm{n}=456\end{array}$ \\
\hline Female, $n(\%)$ & $196(95.1)$ & $430(94.3)$ \\
\hline Age (years), mean (SD) & $39.4(12.04)$ & $38.3(11.77)$ \\
\hline Weight (kg), mean (SD) & $70.0(20.27)$ & $68.4(17.67)$ \\
\hline \multicolumn{3}{|l|}{ Enrolment by region, $n(\%)$} \\
\hline USA & $56(27.2)$ & $109(23.9)$ \\
\hline Americas excluding USA & $39(18.9)$ & $101(22.1)$ \\
\hline Western Europe & $14(6.8)$ & $40(8.8)$ \\
\hline Eastern Europe & $48(23.3)$ & $112(24.6)$ \\
\hline Asia & $49(23.8)$ & $94(20.6)$ \\
\hline \multicolumn{3}{|l|}{ Ethnicity, $n(\%)$} \\
\hline Hispanic or Latino & $59(28.6)$ & $135(29.6)$ \\
\hline Not Hispanic or Latino & $147(71.4)$ & $321(70.4)$ \\
\hline Disease duration (years), median (range) & $4.8(1,33)$ & $4.5(0,35)$ \\
\hline SELENA-SLEDAI, mean (SD) ${ }^{\mathrm{a}}$ & $5.8(3.90)$ & $10.4(3.16)$ \\
\hline \multicolumn{3}{|l|}{ SELENA-SLEDAI organ involvement, $n(\%)$} \\
\hline Mucocutaneous & $108(52.4)$ & $397(87.1)$ \\
\hline Musculoskeletal & $54(26.2)$ & $367(80.5)$ \\
\hline Immunological & $151(73.3)$ & $349(76.5)$ \\
\hline Renal & $24(11.7)$ & $42(9.2)$ \\
\hline Haematological & $10(4.9)$ & $30(6.6)$ \\
\hline Vascular & $7(3.4)$ & $39(8.6)$ \\
\hline Cardiovascular and respiratory & $4(1.9)$ & $20(4.4)$ \\
\hline Constitutional & 0 & $6(1.3)$ \\
\hline CNS & $1(0.5)$ & $7(1.5)$ \\
\hline \multicolumn{3}{|l|}{ BILAG organ domain involvement ${ }^{\mathrm{b}}, n(\%)$} \\
\hline$\geq 1 \mathrm{~A}$ or $2 \mathrm{~B}$ & $42(20.4)$ & $314(68.9)$ \\
\hline$\geq 1 \mathrm{~A}$ & $5(2.4)$ & $69(15.1)$ \\
\hline$\geq 1 \mathrm{~B}$ & $123(59.7)$ & $405(88.8)$ \\
\hline No A or B & $79(38.3)$ & $26(5.7)$ \\
\hline PGA, mean (SD) & $0.8(0.55)$ & $1.6(0.41)^{\mathrm{c}}$ \\
\hline Anti-dsDNA $\geq 30 \mathrm{IU} / \mathrm{mL}, n(\%)$ & $126(61.8)$ & $333(73.0)$ \\
\hline Low C3 ( < 90 mg/dL), $n(\%)$ & $91(44.6)$ & $201(44.1)$ \\
\hline Low C4 ( < $10 \mathrm{mg} / \mathrm{dL}), n(\%)$ & $45(22.1)$ & $119(26.1)$ \\
\hline FACIT-Fatigue, mean (SD) & $36.5(11.45)$ & $32.2(12.01)^{\mathrm{d}}$ \\
\hline \multicolumn{3}{|l|}{ Medications, $n(\%)$} \\
\hline Corticosteroid only & $23(11.2)$ & $44(9.6)$ \\
\hline Antimalarial only & $11(5.3)$ & $31(6.8)$ \\
\hline Immunosuppressant only & $4(1.9)$ & $9(2.0)$ \\
\hline Corticosteroid and antimalarial only & $72(35.0)$ & $172(37.7)$ \\
\hline Corticosteroid and immunosuppressant only & $38(18.4)$ & $70(15.4)$ \\
\hline Immunosuppressant and antimalarial only & $14(6.8)$ & $12(2.6)$ \\
\hline Corticosteroid, immunosuppressant and antimalarial & $41(19.9)$ & $112(24.6)$ \\
\hline
\end{tabular}

Baseline was defined as the last assessment prior to the first dose of belimumab; for the belimumab group this was day 0 of the double-blind phase and for the placebo-to-belimumab group this was week 52 of the double-blind phase.

${ }^{a}$ Among all patients; however, 55 patients in the placebo-to-belimumab group and 2 patients in the belimumab group had a baseline SELENA-SLEDAI score $<4$.

${ }^{\mathrm{b}}$ Patients may be included in more than one category.

${ }^{\mathrm{c}} n=455$.

${ }^{\mathrm{d}} n=454$

BILAG: British Isles Lupus Assessment Group; C3: complement 3; C4: complement 4; CNS: central nervous system; dsDNA: double-stranded DNA; FACIT: Functional Assessment of Chronic Illness Therapy; PGA: Physician's Global Assessment; SC: subcutaneous; SD: standard deviation; SELENA-SLEDAI: Safety of Estrogens in Lupus Erythematosus National Assessment-Systemic Lupus Erythematosus Disease Activity Index; SLE: systemic lupus erythematosus.

respiratory failure occurred in a 37-year-old female in the belimumab group, who developed pneumonia 393 days after receiving the first dose of belimumab $200 \mathrm{mg}$ SC.
Clinical laboratory evaluations

The percentage of patients with worsening $(\geq 2$ grade) of clinical laboratory parameters (haematology, liver function, electrolyte parameters, all other 
Table 2 Summary of adverse events

\begin{tabular}{|c|c|c|}
\hline & $\begin{array}{l}\text { Placebo-to-belimumab } \\
200 \mathrm{mg} \mathrm{SC} \mathrm{n}=206\end{array}$ & $\begin{array}{l}\text { Belimumab } 200 \mathrm{mg} \\
\mathrm{SC} \mathrm{n}=456\end{array}$ \\
\hline Any event, $n(\%)$ & $106(51.5)$ & $220(48.2)$ \\
\hline \multicolumn{3}{|l|}{ AEs by system organ class ${ }^{\mathrm{a}}, n(\%)$} \\
\hline Infections and infestations & $61(29.6)$ & $129(28.3)$ \\
\hline Gastrointestinal disorders & $14(6.8)$ & $41(9.0)$ \\
\hline Musculoskeletal and connective tissue disorders & $19(9.2)$ & $36(7.9)$ \\
\hline Nervous system disorders & $20(9.7)$ & $26(5.7)$ \\
\hline Skin and subcutaneous tissue disorders & $20(9.7)$ & $20(4.4)$ \\
\hline General disorders and administration site conditions & $14(6.8)$ & $25(5.5)$ \\
\hline \multicolumn{3}{|l|}{ AEs by preferred term ${ }^{\mathrm{b}}, n(\%)$} \\
\hline Viral upper respiratory tract infection & $9(4.4)$ & $17(3.7)$ \\
\hline Arthralgia & $7(3.4)$ & $11(2.4)$ \\
\hline Urinary tract infection bacterial & $2(1.0)$ & $15(3.3)$ \\
\hline Nasopharyngitis & $9(4.4)$ & $7(1.5)$ \\
\hline $\mathbf{S A E s}^{\mathrm{c}}, n(\%)$ & $14(6.8)$ & $25(5.5)$ \\
\hline Infections and infestations & $7(3.4)$ & $10(2.2)$ \\
\hline AEs resulting in study discontinuation, $n(\%)$ & $5(2.4)$ & $12(2.6)$ \\
\hline \multicolumn{3}{|l|}{ AEs of special interest, $n(\%)$} \\
\hline Malignancies & 0 & 0 \\
\hline Post-injection systemic reactions ${ }^{\mathrm{d}}$ & $9(4.4)$ & $12(2.6)$ \\
\hline Serious & 0 & 0 \\
\hline Serious delayed non-acute hypersensitivity reactions ${ }^{\mathrm{d}}$ & 0 & 0 \\
\hline All infections of special interest & $7(3.4)$ & $10(2.2)$ \\
\hline Serious & $3(1.5)$ & $2(0.4)$ \\
\hline Opportunistic infections $^{\mathrm{d}}$ & $2(1.0)$ & $1(0.2)$ \\
\hline Serious & $2(1.0)$ & $1(0.2)$ \\
\hline Herpes zoster & $6(2.9)$ & $6(1.3)$ \\
\hline Serious & $2(1.0)$ & 0 \\
\hline Sepsis & 0 & $2(0.4)$ \\
\hline Serious & 0 & $1(0.2)$ \\
\hline Depression & $4(1.9)$ & $8(1.8)$ \\
\hline Serious & 0 & $1(0.2)$ \\
\hline Serious suicidal ideation/self injury ${ }^{\mathrm{d}}$ & 0 & $1(0.2)$ \\
\hline Suicidal behaviour $^{\mathrm{d}}$ & 0 & $1(0.2)$ \\
\hline Deaths, $n(\%)$ & $1(0.5)$ & $1(0.2)$ \\
\hline
\end{tabular}

${ }^{\mathrm{a}} \mathrm{AEs}$ that occurred in $\geq 5 \%$ of patients in either treatment group are listed.

${ }^{b}$ AEs that occurred in $\geq 3 \%$ of patients in either treatment group are listed.

'SAEs that occurred in $>2 \%$ of patients in either treatment group are listed.

${ }^{\mathrm{d}}$ Per GlaxoSmithKline adjudication.

AEs: adverse events; SAEs: serious AEs; SC: subcutaneous.

chemistries and urinalysis) from baseline was low $(<4 \%$ of all patients) (Supplementary Table 1). Overall, $0.5 \%$ of patients in the placeboto-belimumab group and $0.4 \%$ of patients in the belimumab group had a grade 0 to grade 2 change in immunoglobulin G.

\section{Immunogenicity}

Three $(0.5 \%)$ patients $(2[1.2 \%]$ in the placebo-tobelimumab group and $1[0.3 \%]$ in the belimumab group) developed circulating anti-belimumab antibodies during the open-label phase, which subsequently resolved in each patient. None of these patients experienced an SAE; one reported a post-injection systemic reaction (rash), which was mild in severity and was not a hypersensitivity reaction.

\section{Efficacy}

SRI response

At week $24,16.1 \%(23 / 143)$ of patients in the placebo-to-belimumab group and $76.3 \%(332 / 435)$ of patients in the belimumab group achieved an SRI4 response (Figure 3).

$\mathrm{A} \geq 4$-point improvement in SELENA-SLEDAI, no worsening in PGA and no new BILAG $1 \mathrm{~A} / 2 \mathrm{~B}$ score were reported for $17.5 \%$ (25/143), $87.4 \%$ $(125 / 143)$, and $93.7 \%(134 / 143)$ of the placebo-tobelimumab group, respectively, and $79.3 \%$ (345/ 


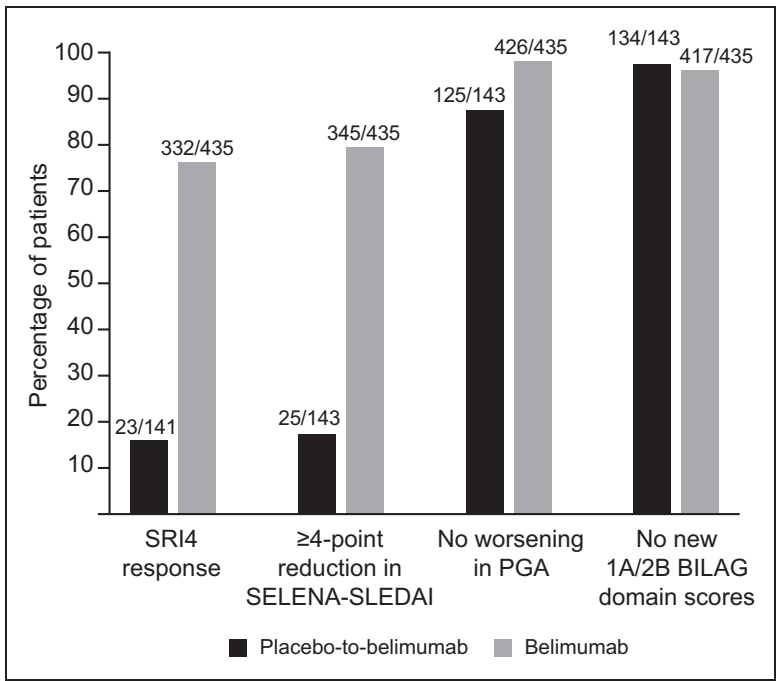

Figure 3 Systemic lupus erythematosus Responder Index response at week 24 .

BILAG: British Isles Lupus Assessment Group; PGA: Physician's Global Assessment; SELENA-SLEDAI: Safety of Estrogens in Lupus Erythematosus National AssessmentSystemic Lupus Erythematosus Disease Activity Index; SRI: Systemic lupus erythematosus Responder Index.

435), $97.9 \%(426 / 435)$ and $95.9 \%(417 / 435)$ of the belimumab group, respectively.

\section{SFI flare}

During the open-label phase, $1.0 \%(2 / 206)$ of patients in the placebo-to-belimumab group and $2.6 \%(12 / 456)$ of patients in the belimumab group had a severe flare. Among these patients, median (range) time to first severe flare was $168.5(167,170)$ days in the placebo-to-belimumab group and 169.0 $(117,194)$ days in the belimumab group. In total, $18.4 \%(38 / 206)$ of patients in the placebo-to-belimumab group and $12.7 \%(58 / 456)$ in the belimumab group had any (mild, moderate or severe) flare during the open-label extension.

\section{Renal flares}

Renal flares (mild, moderate or severe) occurred in $1.5 \%(3 / 206)$ of patients in the placebo-to-belimumab group and $3.5 \%(16 / 456)$ of patients in the belimumab group. Among patients with baseline proteinuria $>0.5 \mathrm{~g} / 24$ hours, fewer patients in the placebo-to-belimumab group $(6.3 \%, 2 / 32)$ had a renal flare compared with the belimumab group $(9.3 \%, 7 / 75)$.

\section{Prednisone use}

Among patients receiving a baseline prednisone dose $>7.5 \mathrm{mg} /$ day, $9.8 \%(10 / 102)$ in the placeboto-belimumab group and $24.4 \%(67 / 275)$ in the belimumab group had a dose reduction to $\leq 7.5 \mathrm{mg}$ /day by week 24 . In patients with baseline prednisone dose $\leq 7.5 \mathrm{mg} /$ day, $1.9 \%(2 / 104)$ of the placebo-to-belimumab group and 5.0\% (9/181) of the belimumab group had a dose increase to $>7.5 \mathrm{mg} /$ day.

\section{Biomarkers}

Among patients with anti-dsDNA antibodies at baseline, the median (25th, 75th percentile) reductions at week 24 were $-35.6 \%(-51.9 \%,-9.2 \%$; $n=121)$ in the placebo-to-belimumab group and $-62.8 \%(-77.9 \%,-39.0 \% ; n=326)$ in the belimumab group. Of the patients who were anti-dsDNA antibody-positive at baseline, $13.2 \%(16 / 121)$ in the placebo-to-belimumab group and 21.5\% (70/326) in the belimumab group were anti-dsDNA antibody-negative at week 24 (Table 3 ).

Among patients with low C3 levels at baseline $(<90 \mathrm{mg} / \mathrm{dL})$, the mean (SD) percent changes in C3 from baseline to week 24 were $16.7 \%$ (23.46; $n=88)$ and $29.1 \%(38.11 ; n=198)$ for the placebo-to-belimumab and belimumab groups, respectively. In patients with low $\mathrm{C} 4$ levels at baseline $(<10 \mathrm{mg} / \mathrm{dL})$, the mean (SD) percent changes in $\mathrm{C} 4$ levels were $50.6 \%(82.69 ; n=43)$ and $77.6 \%$ $(83.44 ; n=117)$ for the placebo-to-belimumab and belimumab groups, respectively (Table 3 ).

From baseline to week 24, there were greater reductions in levels of $\mathrm{CD} 19+, \mathrm{CD} 20+$, naïve $(\mathrm{CD} 19+/ \mathrm{CD} 20+/ \mathrm{CD} 27-)$ and activated (CD19+/ $\mathrm{CD} 20+/ \mathrm{CD} 69+) \mathrm{B}$ cells among patients in the belimumab group compared with the placebo-tobelimumab group (Table 3 ).

\section{Discussion}

This 6-month extension of the BLISS-SC study examined the safety, tolerability and efficacy of belimumab SC in patients with SLE and demonstrates comparable safety and efficacy to that observed in the 52-week, double-blind phase of the study. 7

In this open-label phase, belimumab was well tolerated over 24 weeks of treatment, with an overall incidence rate of AEs similar to that observed in the 52-week double-blind phase. ${ }^{7}$ Most AEs were of mild or moderate intensity. The overall rate of treatment discontinuation due to AEs was lower in the open-label phase compared with that reported in the 52-week double-blind phase. The rates of AESIs were low and there were no discontinuations due to infections of special interest. The 
Table 3 Changes in biomarker measures from baseline at week 24

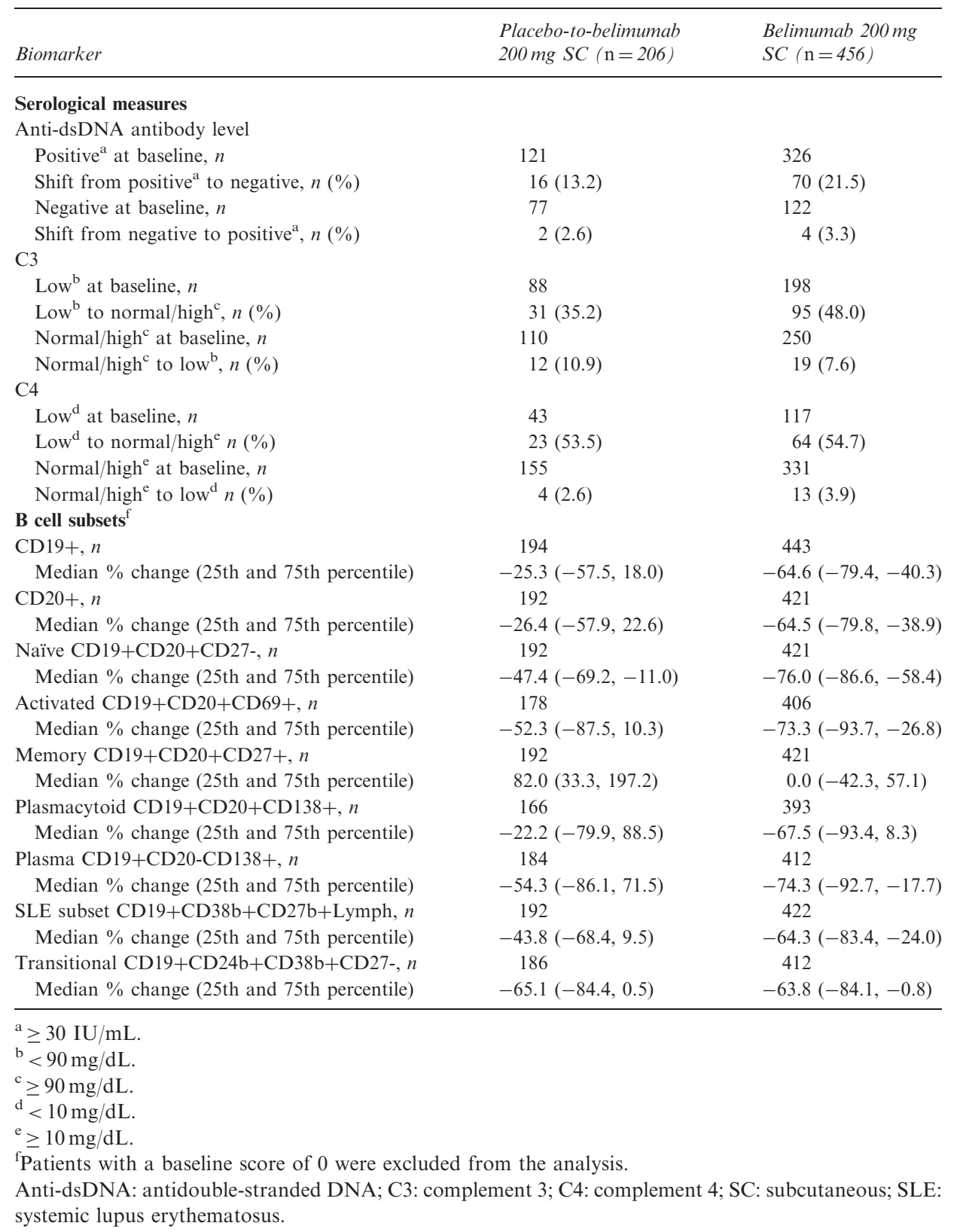

safety profile of belimumab SC in this study is in line with that demonstrated in the Phase III doubleblind and open-label extension studies of belimumab IV ${ }^{16-18}$ and in clinical practice. ${ }^{19,20}$

The efficacy of belimumab was maintained throughout the open-label phase. An SRI response was achieved by $16.1 \%$ of patients in the placebo-tobelimumab group who received belimumab for 24 weeks, and $76.3 \%$ patients in the belimumab group who received belimumab for a total of 76 weeks. At the end of the double-blind phase (52 weeks), 61.4\% of the belimumab group had an SRI response; ${ }^{7}$ therefore, during the open-label phase, efficacy was maintained or improved with ongoing exposure to belimumab. These results suggest that 24 weeks of belimumab may not be sufficient for a complete response. However, the higher SRI response rate observed in the belimumab group compared with the placebo-to-belimumab group could also be due to the lower baseline SELENA-SLEDAI in the placebo-to-belimumab group compared with the belimumab group, which made it more difficult to demonstrate improvement. Furthermore, since the SRI response requires a four-point reduction in SELENA-SLEDAI and 55 patients in the placebo-to-belimumab group had a baseline 
SELENA-SLEDAI score of $<4$ at the start of the open-label extension, they were excluded from this analysis. The rate of severe flares was low across the entire study population. Among patients receiving a baseline prednisone dose $>7.5 \mathrm{mg}$ /day, $9.8 \%$ in the placebo-to-belimumab group and $24.4 \%$ in the belimumab group had a dose reduction to $\leq 7.5 \mathrm{mg}$ /day by week 24; this is concordant with Phase III double-blind studies of belimumab IV. ${ }^{16,18}$

There are several limitations to the interpretation of these data. First, there was no placebo control arm during the open-label extension; therefore, results cannot be definitively attributed to belimumab. Moreover, the baseline clinical characteristics for the placebo-to-belimumab group were different from those for the belimumab group, due to the difference in timing of the baselines and differences in the inclusion criteria of the two phases of the study. The duration of exposure to belimumab differed between the two treatment groups; therefore, no comparisons are made between the groups. Patients who were randomized to placebo had lower disease activity at baseline for the open-label phase compared with baseline in the double-blind phase, reflecting improvements in disease activity during the double-blind phase. $^{7}$ Additionally, patients were required to have a SELENA-SLEDAI score $\geq 8$ to be eligible to enter into the 52-week, double-blind phase, whereas there was no such criterion for inclusion in the open-label phase. As such, 55 patients in the placebo-to-belimumab group had a baseline SELENA-SLEDAI score of $\leq 4$ and were excluded from the SRI analyses; in the belimumab group there were four patients who were excluded from the analyses due to having a baseline SELENA-SLEDAI score of $\leq 4$. There may have been some selection bias as patients who completed the double-blind phase and were therefore eligible to enter the extension phase were those who responded to or tolerated belimumab or placebo in the double-blind phase. Patients who maintained disease control or improved while receiving placebo plus SoC in the double-blind phase may represent patients with stable, less active disease. Furthermore, the treatment groups received belimumab for different durations; therefore, statistical comparisons between the two groups cannot be made.

In summary, belimumab $200 \mathrm{mg}$ SC as add-on therapy to SoC was well tolerated in patients with SLE during this 24-week extension phase of the BLISS-SC study. The safety profile was in line with that observed during the double-blind phase, with no increase in AEs and SAEs, and no new safety signals. Moreover, the efficacy of belimumab was maintained across the extension phase.
Together with the ease of use, financial and timesaving benefits of the SC route of administration, ${ }^{7,10,12}$ the results of this study suggest that belimumab SC could provide a desirable additional treatment option for patients with SLE.

\section{Key messages}

- The safety and tolerability of up to 76 weeks' treatment with subcutaneous belimumab is acceptable.

- The efficacy of subcutaneous belimumab was maintained during this open-label extension phase.

- Subcutaneous belimumab will provide an additional treatment option for patients with systemic lupus erythematosus.

\section{Acknowledgments}

The authors would like to thank all the investigators and patients for their participation in the study. Shweta Vadnerkar, PhD, and Katie White, $\mathrm{PhD}$, of Fishawack Indicia Ltd, provided medical writing support, funded by GSK.

\section{Declaration of conflicting interests}

The authors declared the following potential conflicts of interest with respect to the research, authorship and/or publication of this article: A.D. has received consultancy payments from GSK, Pfizer, AstraZeneca, Celgene, Eli Lilly and Baxalta; A.H., J.G., D.B., D.R. and D.G. are employees of GSK and hold shares in the company; A.S. has received consultancy payments from GSK; M.S. has received consultancy payments from GSK, Pfizer, Epirus, Samsung Bioepis and Janssen Pharmaceutical Products L.P.; N.L.F. was an employee of GSK and held shares in GSK at the time of the study; W.S. has received consultancy or research grants from GSK, Celgene, Janssen Research \& Development, Pfizer and Sanofi-Aventis Pharmaceutical; and C.K. is an employee of Parexel and holds shares in GSK.

\section{Funding}

The authors disclosed receipt of the following financial support for the research, authorship 
and/or publication of this article: this study was sponsored by GlaxoSmithKline (study number: BEL112341).

\section{Availability of study materials}

The study protocol and clinical study report are freely available from https://gsk-clinicalstudyregis ter.com/

\section{References}

1 Baker KP, Edwards BM, Main SH, et al. Generation and characterization of LymphoStat-B, a human monoclonal antibody that antagonizes the bioactivities of B lymphocyte stimulator. Arthritis Rheum 2003; 48: 3253-3265.

2 Hase H, Kanno Y, Kojima M, et al. BAFF/BLyS can potentiate B-cell selection with the B-cell coreceptor complex. Blood 2004; 103 : 2257-2265.

3 Vilas-Boas A, Morais SA, Isenberg DA. Belimumab in systemic lupus erythematosus. RMD Open 2015; 1: e000011.

4 European Medicines Agency. Summary of product characteristics. http://www.ema.europa.eu/ema/ (2011, accessed 5 May 2017).

5 US Food and Drug Administration. FDA approves Benlysta to treat lupus. https://wayback.archive-it.org/7993/20170112024037/ http://www.fda.gov/NewsEvents/Newsroom/PressAnnouncements/ ucm246489.htm (2011, accessed 14 May 2018).

6 Gatto M, Iaccarino L, Zen M, et al. When to use belimumab in SLE. Expert Rev Clin Immunol 2017; 13: 737-740.

7 Stohl W, Schwarting A, Okada M, et al. Efficacy and safety of subcutaneous belimumab in systemic lupus erythematosus: A fiftytwo-week randomized, double-blind, placebo-controlled study. Arthritis Rheumatol 2017; 69: 1016-1027.

8 Cai WW, Fiscella M, Chen C, et al. Bioavailability, pharmacokinetics, and safety of belimumab administered subcutaneously in healthy subjects. Clin Pharmacol Drug Dev 2013; 2: 349-357.
9 Yapa SW, Roth D, Gordon D, et al. Comparison of intravenous and subcutaneous exposure supporting dose selection of subcutaneous belimumab systemic lupus erythematosus Phase 3 program. Lupus 2016; 25: 1448-1455.

10 Jin JF, Zhu LL, Chen M, et al. The optimal choice of medication administration route regarding intravenous, intramuscular, and subcutaneous injection. Patient Prefer Adherence 2015; 9: 923-942.

11 Stoner KL, Harder H, Fallowfield LJ, et al. Intravenous versus subcutaneous drug administration. Which do patients prefer? A systematic review. Patient 2014; 8: 145-153.

12 Chilton F, Collett RA. Treatment choices, preferences and decision-making by patients with rheumatoid arthritis. Musculoskeletal Care 2008; 6: 1-14.

13 Hochberg MC. Updating the American College of Rheumatology revised criteria for the classification of systemic lupus erythematosus. Arthritis Rheum 1997; 40: 1725.

14 World Medical Association. WMA Declaration of Helsinkiethical principles for medical research involving human subjects. https://www.wma.net/wp-content/uploads/2016/11/DoH-Oct2008. pdf (2008, accessed May 15, 2018).

15 Furie RA, Petri MA, Wallace DJ, et al. Novel evidence-based systemic lupus erythematosus responder index. Arthritis Rheum 2009; 61: 1143-1151.

16 Navarra SV, Guzman RM, Gallacher AE, et al. Efficacy and safety of belimumab in patients with active systemic lupus erythematosus: a randomised, placebo-controlled, phase 3 trial. Lancet 2011; 377: 721-731.

17 Bruce IN, Urowitz M, van Vollenhoven R, et al. Long-term organ damage accrual and safety in patients with SLE treated with belimumab plus standard of care. Lupus 2016; 25: 699-709.

18 Furie R, Petri M, Zamani O, et al. A phase III, randomized, placebo-controlled study of belimumab, a monoclonal antibody that inhibits B lymphocyte stimulator, in patients with systemic lupus erythematosus. Arthritis Rheum 2011; 63: 3918-3930.

19 Iaccarino L, Bettio S, Reggia R, et al. Effects of belimumab on flare rate and expected damage progression in patients with active systemic lupus erythematosus. Arthritis Care Res (Hoboken) 2017; 69: 115-123.

20 Collins CE, Dall'Era M, Kan H, et al. Response to belimumab among patients with systemic lupus erythematosus in clinical practice settings: 24-month results from the OBSErve study in the USA. Lupus Sci Med 2016; 3: e000118. 